\title{
Time is on My (Our) Side
}

\section{Carlo Melloni*}

Department of Surgical, Oncological and Oral Sciences, Plastic and Reconstructive Surgery Unit, University of Palermo, Italy

*Corresponding author: Carlo Melloni, M.D., Department of Surgical, Oncological and Oral Sciences, Plastic and Reconstructive Surgery Unit, University of Palermo, Italy, Tel: +39 091 6554034; Fax: +39 091 6553720; E-mail: carlomelloni.unipa@gmail.com

Received date: February 13, 2015, Accepted date: February 14, 2015, Published date: February 17, 2015

Copyright: (C) 2015 Melloni C. This is an open-access article distributed under the terms of the Creative Commons Attribution License, which permits unrestricted use, distribution, and reproduction in any medium, provided the original author and source are credited.

\section{Abstract}

The surgeon is obviously considered as an important prognostic factor for patient's outcome and several studies investigated on quality and outcomes of cares of patients that often look for expert surgical teams and centers of excellence.

Be a team of excellence means high-volume practice of complex surgery but there is also sufficient evidence from the literature to indicate that equally good results can be obtained in smaller regional hospitals by some surgeons who only operate on a low volume of cases.

What does it mean? Is it possible for residents to reach a high level of expertise operating on low volume of cases and without enough resources?

\section{Editorial}

The Editorial, "Time is on my (our) side" is based on the consistent literature evaluating the relationship between outcomes and case volume per surgeon [1], distinguishing expert surgeons from residents [2].

I choose this title, based on a famous song by The Rolling Stones, to underline the role of residents and tutors on the educational programs of our academic institutions.

The surgeon is obviously considered as an important prognostic factor for patient's outcome and several studies investigated on quality and outcomes of cares of patients that often look for expert surgical teams and centers of excellence.

Be a team of excellence means high-volume practice of complex surgery but there is also sufficient evidence from the literature to indicate that equally good results can be obtained in smaller regional hospitals by some surgeons who only operate on a low volume of cases.

What does it mean? Is it possible for residents to reach a high level of expertise operating on low volume of cases and without enough resources?

Today, if we look back at the past of high-skilled-surgeons we can see that, at the beginnings of their experience, they too started out with low volumes of cases. Through passion, personal and relentless efforts, however, they developed the expertise for which they are now famous but they also attribute a pivotal role to their mentor, the one who guided them through their training.
Professional networks, subscriptions in scientific societies, stages and training experience in a specific field will add the plus to our formation but it can be considered only as the "tip of the iceberg"; I strongly believe that it is essential for us, as residents in training, to find out who want to transmit his/her expertise.

Transmission of expertise in a mentor-to-pupils way both in formal teaching and in surgical guidance is the key to gain or lose passion in our work. Academic centers have the opportunity to consolidate their educational role by looking at the quality of teaching and not only at the volume of procedures. Be an academic center of excellence means giving the right opportunities to students and residents, leaving them free to learn techniques and to practice as surgeons directly involved in patients study, surgical planning and post-operative management but always guided and tutored.

It is generally accepted that plastic surgery performed by young surgeons in training will be less successful and effective than such surgery performed by experienced and well-trained senior surgeons but there is sufficient evidence from the literature that a well-trained and well-supervised resident will obtain results equally as good as his or her supervisor or senior surgeon, without compromising immediate outcome [3]. Supervision of surgeons in training, however, may help to bridge this difference in experience and surgical expertise.

So the best opportunity for a resident to become an expert and skilled surgeon is to find a good mentor, a solid training period, followed by continuing medical education.

So save space and opportunities for your young residents! They will be the surgeons of tomorrow.

\section{References}

1. Matthews HR, Powell DJ, McConkey CC (1986) Effects of surgical experience on the results of resection for oesophageal carcinoma. Br J Surg 73: 621-623.

2. Singh KK1, Aitken RJ (1999) Outcome in patients with colorectal cancer managed by surgical trainees. Br J Surg 86: 1332-1336.

3. Jordan SW, Mioton LM, Smetona J, Aggarwal A, Wang E, et al. (2013) Resident involvement and plastic surgery outcomes: an analysis of 10,356 patients from the American College of Surgeons National Surgical Quality Improvement Program database. Plast Reconstr Surg 131: 763-73. 